\title{
Salivary Gland Sebaceous Lymphadenoma
}

National Cancer Institute

\section{Source}

National Cancer Institute. Salivary Gland Sebaceous Lymphadenoma. NCI Thesaurus.

Code C35555.

A rare, benign epithelial neoplasm composed of irregular proliferating nests and islands of epithelium, including solid and gland-like sebaceous elements, surrounded by lymphoid stroma. Most occur in the parotid gland. Symptoms include a progressively enlarging, painless mass. Sebaceous lymphadenocarcinoma is the malignant counterpart. 